\title{
A Multiscale Workflow for Modelling Ligand Complexes of Zinc Metalloproteins
}

\author{
Zongfan Yanga,b, Rebecca M. Twidalea, Silvia Gervasonia,c,d, Reynier Suardíaz ${ }^{\text {a,e }}$, \\ Charlotte K. Colenso a,b, Eric J. M. Langa , James Spencerb and Adrian J. Mulhollanda* \\ aCentre for Computational Chemistry, School of Chemistry, University of Bristol, Bristol, BS8 1TH, \\ U.K. \\ bSchool of Cellular and Molecular Medicine, University of Bristol, Bristol, BS8 1TD, U.K. \\ cDepartment of Pharmaceutical Sciences, University of Milan, Via Mangiagalli, 25, I-20133 Milano, \\ Italy. \\ dDepartment of Physics, University of Cagliari, S.P. Monserrato-Sestu km 0.700 I-09042, Monserrato \\ (CA), Italy (present address) \\ eDepartamento de Química Física, Facultad de Química, Universidad Complutense, 28040 Madrid, \\ Spain (present address)
}

Supporting Information

Contents

Figure S1. Zinc site coordination distances against simulation time for 200 ps DFTB3 QM/MM MD simulation of the Sfh-I:L-anti-1a complex.

Figure S2. Zinc site coordination distances against simulation steps over 250-step B3LYP-D3BJ based QM/MM geometry optimization of the Sfh-I:L-anti-1a complex without (A) and with (B) diffuse function.

Figure S3. Time-dependence of RMSD values compared to the crystal structure for MM MD simulations of the ACE:L-captopril complex using LJ12-6-4 non-bonded models.

Figure S4. Representative zinc site geometries of the ACE:L-captopril complex after MM MD simulations and QM optimization.

Figure S5. Time-dependence of RMSD values compared to the crystal structure for MM MD simulations of the ACE2:MLN-4760 complex using LJ12-6-4 non-bonded models. 
Figure S6. Representative zinc site geometries of the ACE2:MLN-4760 complexes after MM MD simulations and QM optimization.

Figure S7. Time-dependence of RMSD values compared to the crystal structure for MM MD simulations of the HDAC2:SAHA complex using non-bonded models.

Figure S8. Representative zinc site geometries of the HDAC2:SAHA complex after MM MD simulations and QM optimization.

Figure S9. Time-dependence of RMSD values compared to the crystal structure for MM MD simulations of the IMP1:TGA complex using non-bonded models.

Figure S10. Representative zinc site geometries of the IMP1:TGA complex after MM MD simulations and QM optimization.

Figure S11. Time-dependence of RMSD values compared to the crystal structure for MM MD simulations of the L1:hydrolyzed faropenem complex using LJ12-6-4 non-bonded models.

Figure S12. Representative zinc site geometries of the L1:hydrolyzed faropenem complex after MM MD simulations and QM optimization.

Figure S13. Outcome of DFTB3/MM MD run directly from crystal structures.

Table S1. Zn coordination distance $(\AA ̊)$ of four non-bonded MM models for Sfh-I: L-anti-1a complex.

Table S2. Coordination distances between the zinc ion and its coordinating atoms for QM/MM simulations of the Sfh-I:L-anti-1a complex.

Table S3. Atomic distances $(\AA ̊)$ between the zinc ions and the zinc coordinating atoms for simulations of the ACE inhibitor complex model.

Table S4. Atomic distances $(\AA)$ between the zinc ions and the zinc coordinating atoms for simulations of the ACE2 inhibitor complex model.

Table S5. Zn coordination distance $(\AA ̊)$ of four non-bonded MM models for the HDAC-2:SAHA complex.

Table S6. QM/MM optimized distances ( $)$ between the zinc ion and its coordinating atoms for simulations of the HDAC2:SAHA complex.

Table S7. Zn coordination distance (Å) of four non-bonded MM models for simulations of the IMP1:TGA complex.

Table S8. Representative QM/MM optimized atomic distances ( $\mathrm{Q}$ ) between the zinc ions and the zinc coordinating atoms for simulations of the IMP-1:TGA complex model. 
Table S9. Representative atomic distances $(\AA ̊)$ between the zinc ions and the zinc coordinating atoms for simulations of the L1:Faropenem complex model. 


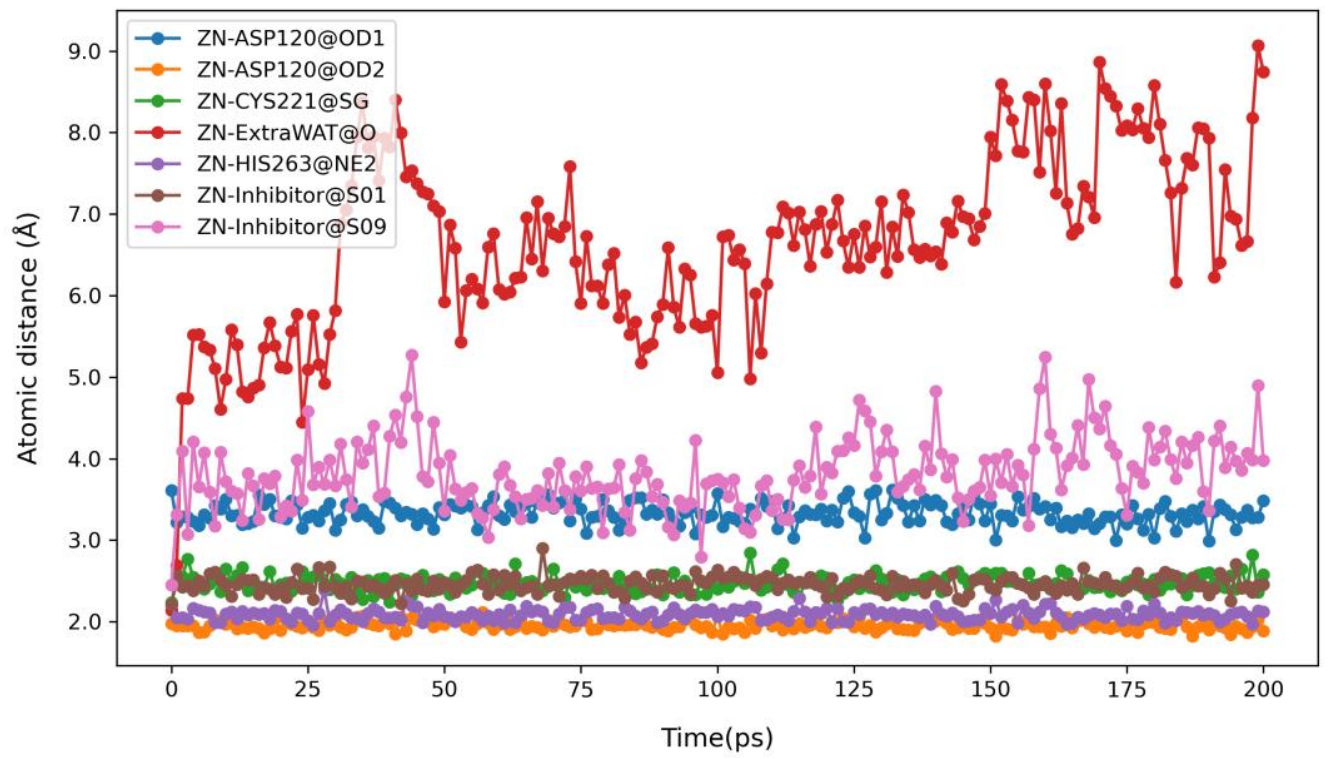

Figure S1. Zinc site coordination distances against simulation time for 200 ps DFTB3 QM/MM MD simulation of the Sfh-I:L-anti-1a complex. All $\mathrm{Zn}^{2+}$ ligation distances are recorded, including water coordination (ZN-ExtraWat@0) added by the MM MD. The major zinc geometry shift happens at the beginning of the QM/MM MD run. Data for each interaction was collected every picosecond, with dots indicating data collection points. 
(A)

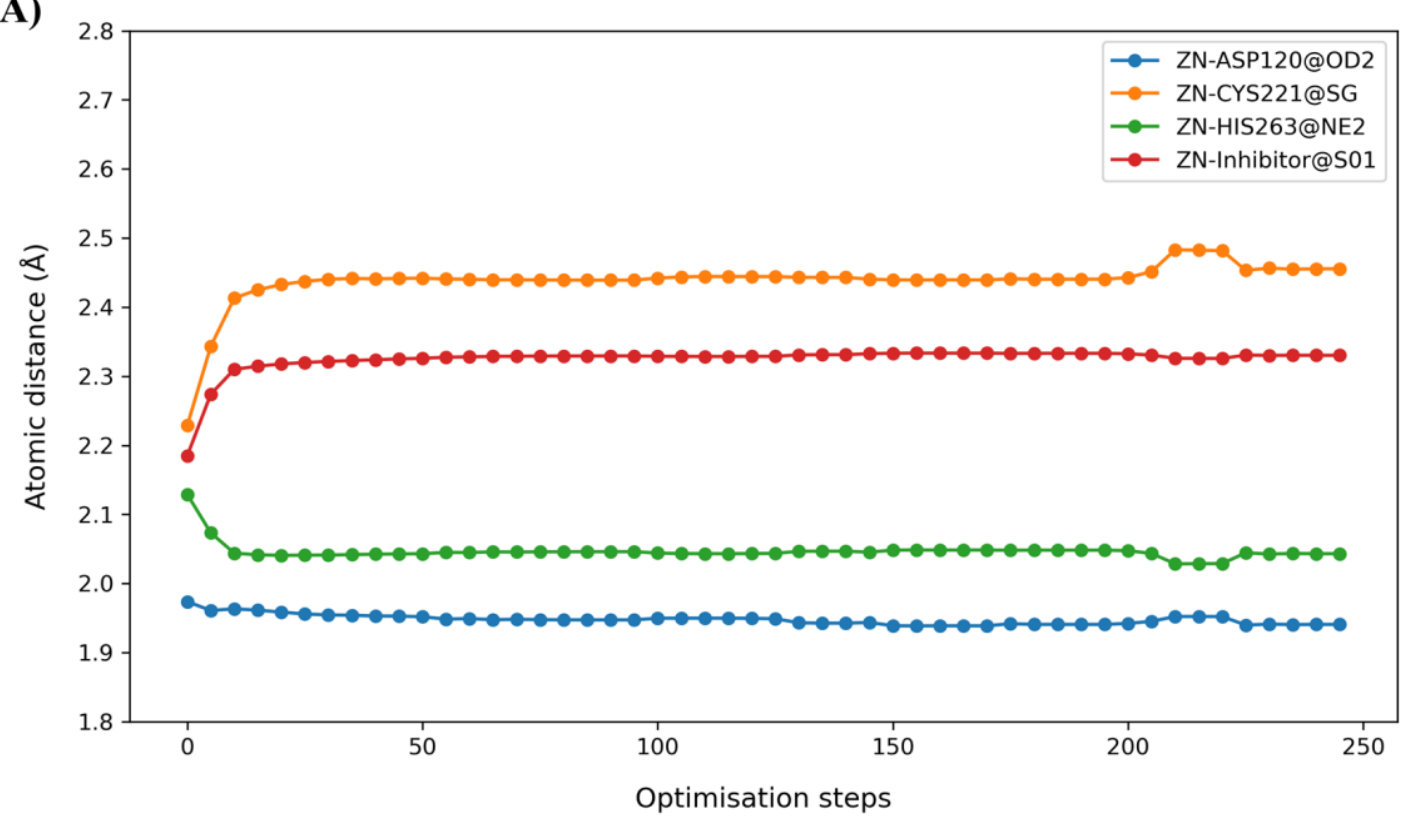

(B)

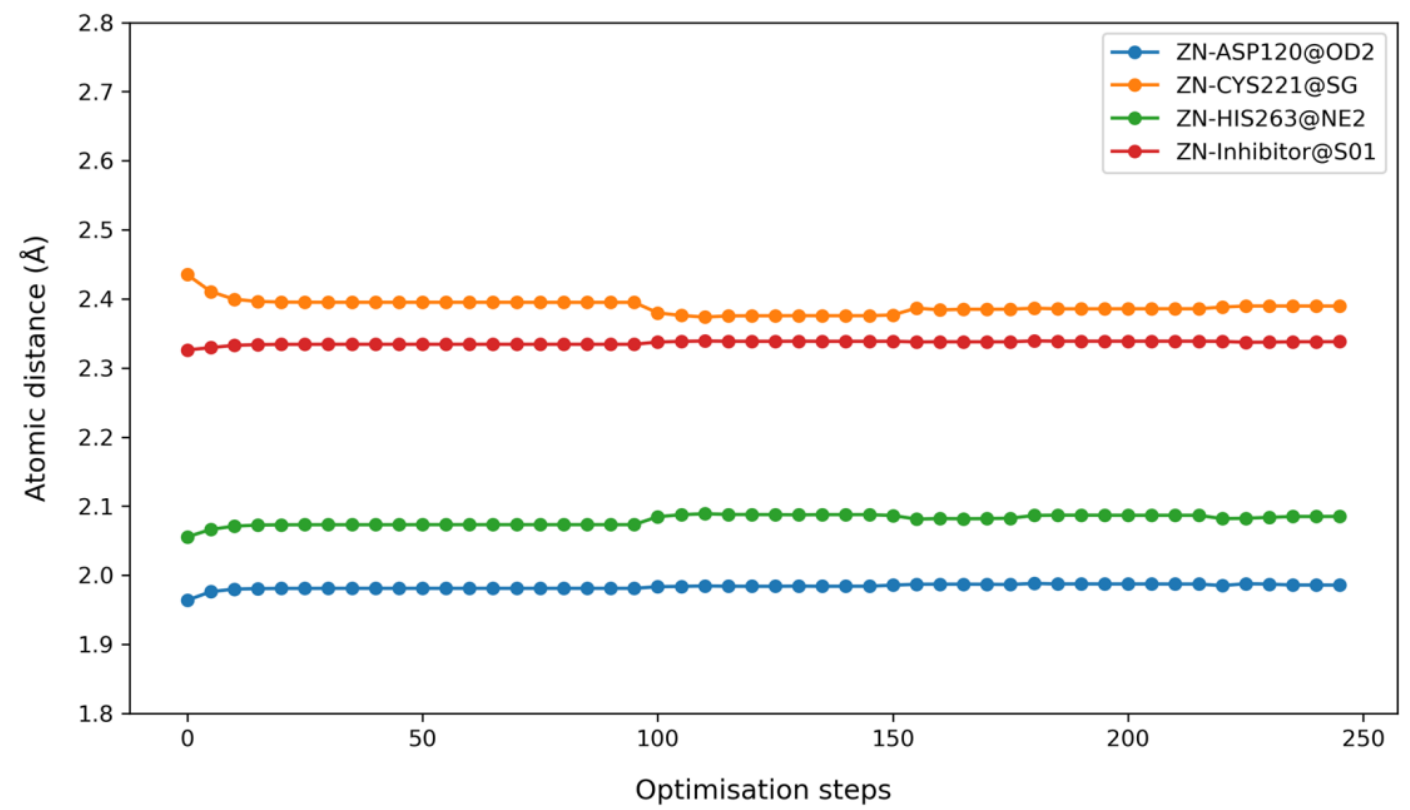

Figure S2. Zinc site coordination distances against simulation steps over 250-step B3LYP-D3BJ based QM/MM geometry optimization of the Sfh-I:L-anti-1a complex without (A) and with (B) diffuse function.

The major zinc geometry shift happens at the beginning of the QM/MM optimization. Data for each interaction was collected every 5 steps, with dots indicating data collection points. 


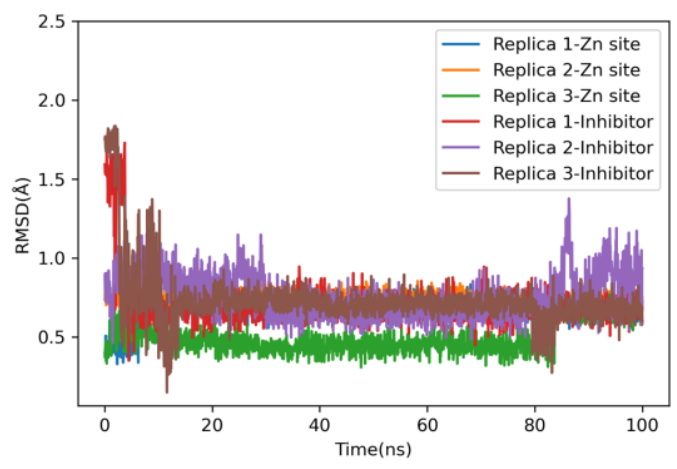

(A)

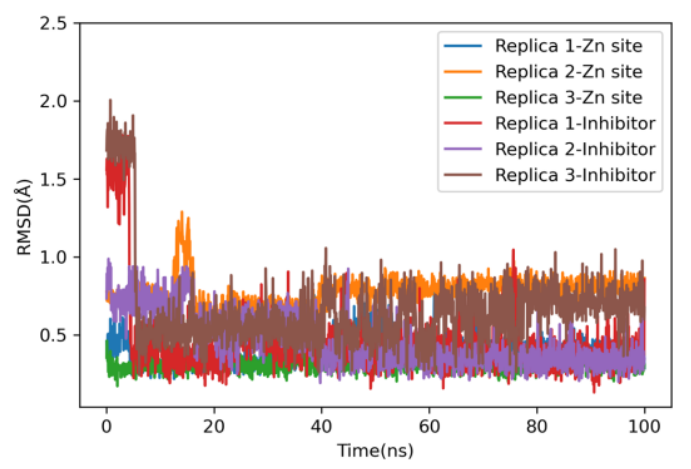

(B)

LJ12-6-4

Figure S3. Time-dependence of RMSD values compared to the crystal structure for MM MD simulations of the ACE:L-captopril complex using LJ12-6-4 non-bonded models. Three replicate simulations were performed for each model and each replicate is $100 \mathrm{~ns}$. The 'Zn site' refers to zinc ions and zinc coordinating residues and 'Inhibitor' refers to the zinc bound inhibitor L-captopril. 


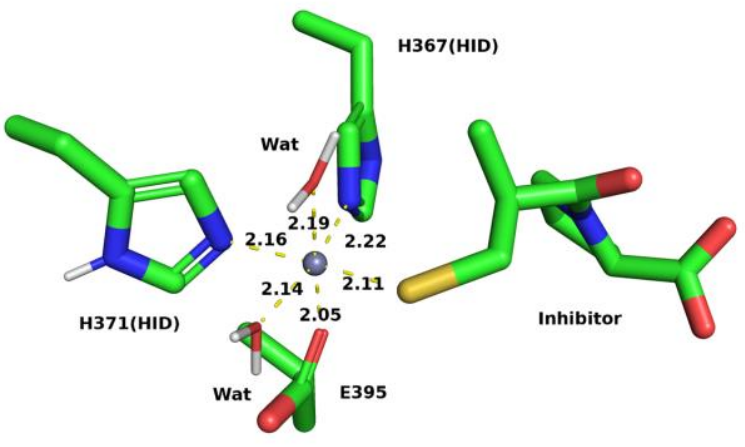

(A)

LJ12-6-4-R

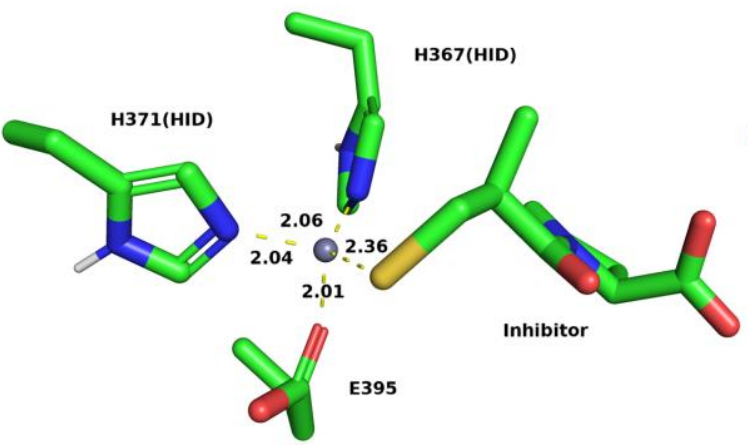

(C)

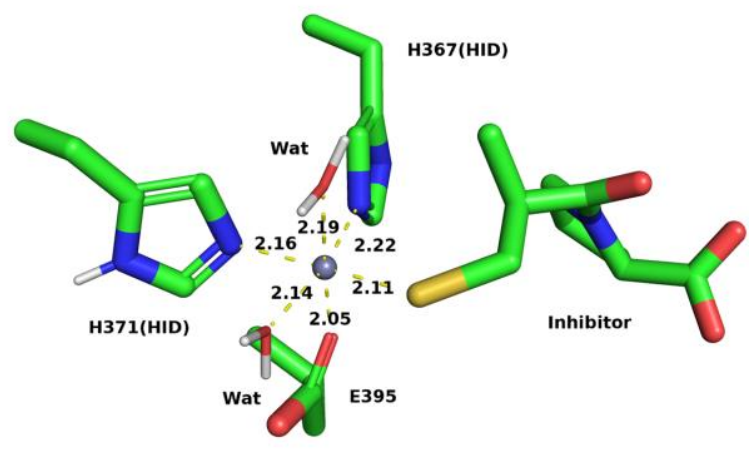

(B)

LJ12-6-4

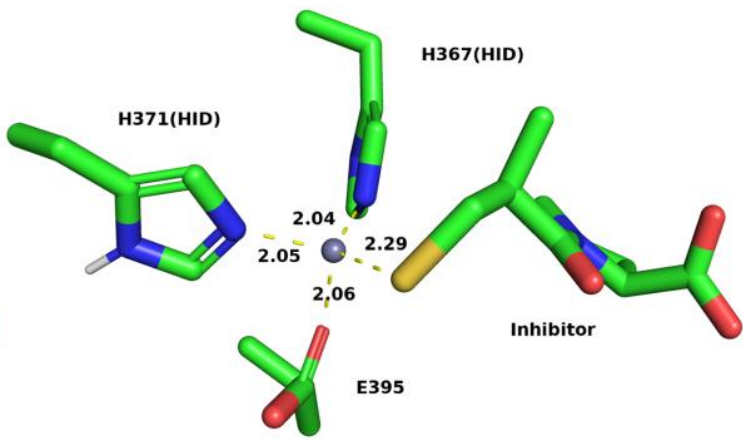

(D)

Figure S4. Representative zinc site geometries of the ACE:L-captopril complex after MM MD simulations and QM optimization. (A-B) Representative zinc site geometries after 100 ns MM MD simulations using LJ12-64 model with (A) and without (B) restraints. (C) Representative zinc geometry after 100 ps DFTB3/MM MD simulation. The extra water molecule was removed. (D) Representative zinc geometry after B3LYP-D3BJ based QM/MM geometry optimization. 


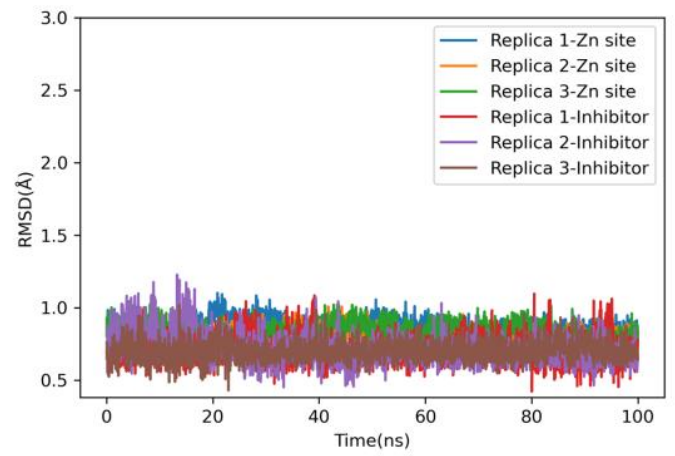

(A)

LJ12-6-4-R

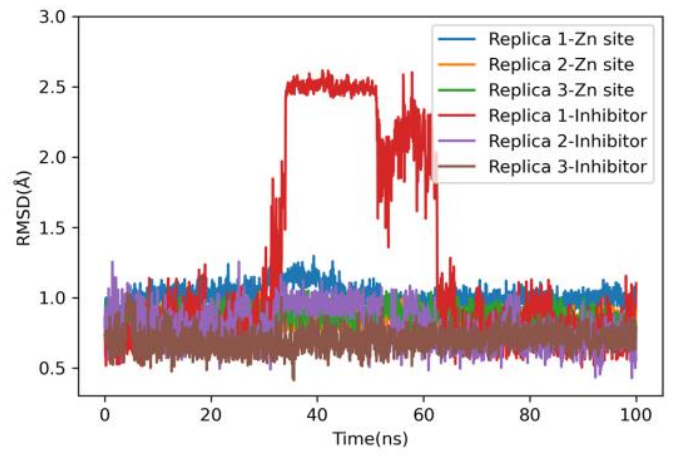

(B)

Figure S5. Time-dependence of RMSD values compared to the crystal structure for MM MD simulations of the ACE2:MLN-4760 complex using LJ12-6-4 non-bonded models. Three replicate simulations were performed for each model and each replicate is $100 \mathrm{~ns}$. The 'Zn site' refers to zinc ions and zinc coordinating residues and 'Inhibitor' refers to the zinc bound inhibitor MLN-4760. 


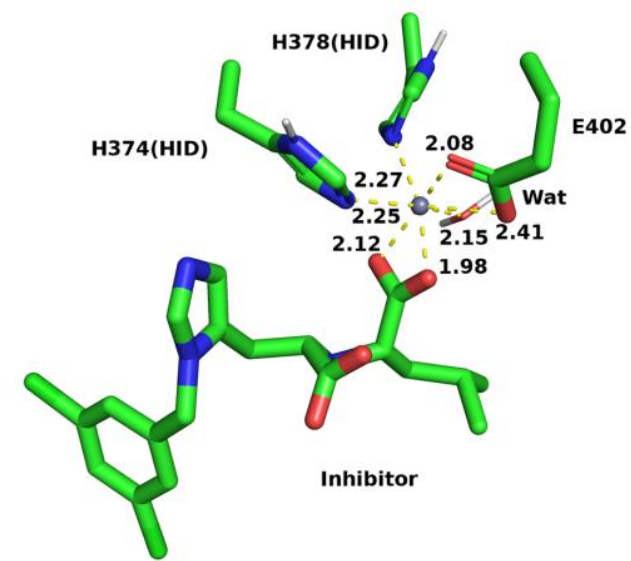

(A)

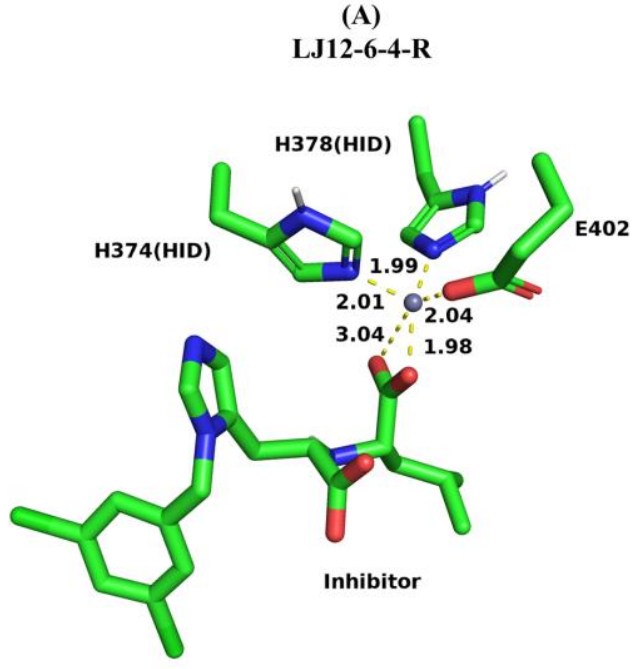

(C)

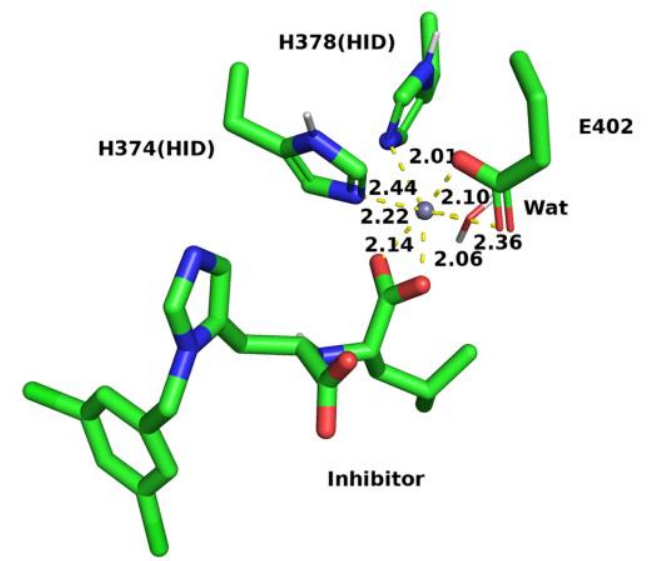

(B)

LJ12-6-4

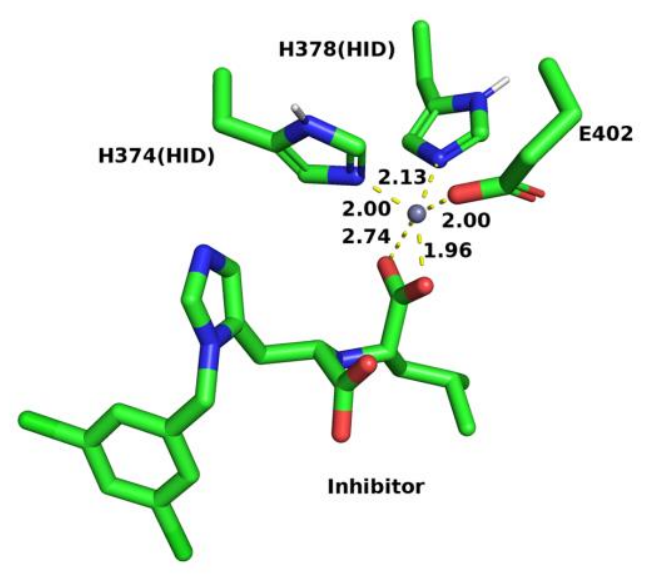

(D)

B3LYP

Figure S6. Representative zinc site geometries of the ACE2:MLN-4760 complexes after MM MD simulations and QM optimization. (A-B) geometries after 100 ns MM MD simulations using LJ12-6-4 model without (A) and with (B) restraints. (C) Representative zinc geometry after 100 ps DFTB3/MM MD simulation. The extra water molecule was removed. (D) Representative zinc geometry after B3LYP-D3BJ based QM/MM geometry optimization. 


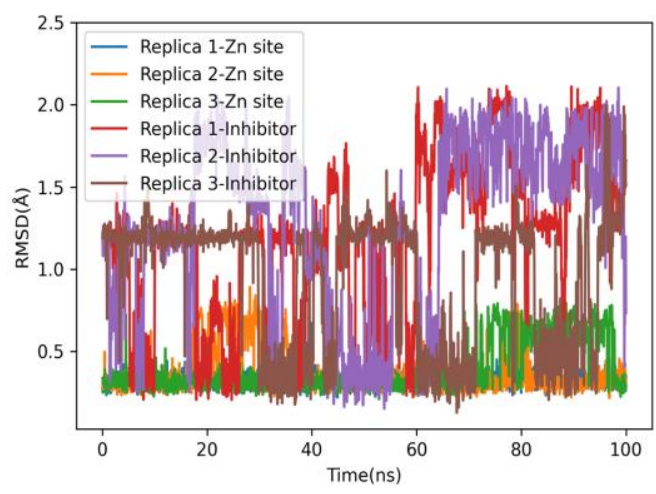

(A)

LJ12-6-4-R

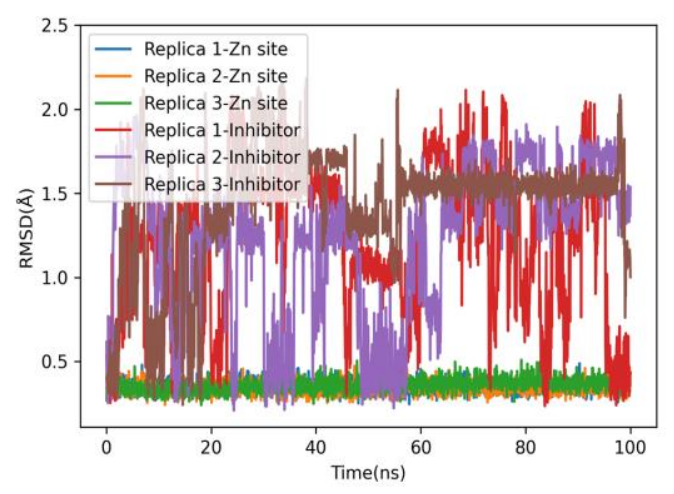

(C)

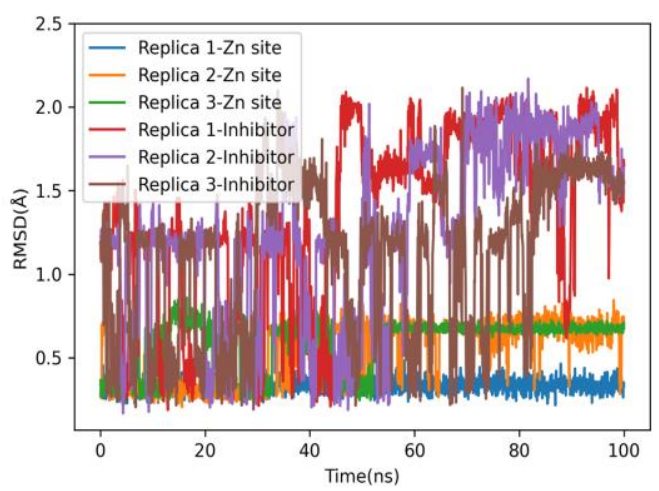

(B)

LJ12-6-4

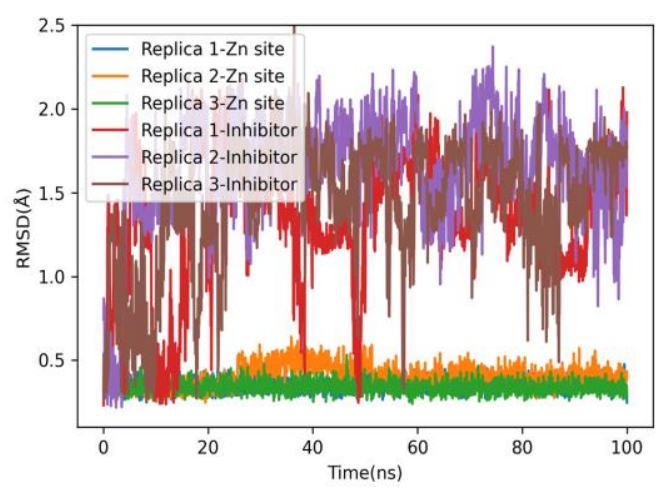

(D)

Figure S7. Time-dependence of RMSD values compared to the crystal structure for MM MD simulations of the HDAC2:SAHA complex using non-bonded models. Three replicate simulations were performed for each model and each replicate is $100 \mathrm{~ns}$. The 'Zn site' refers to zinc ions and zinc coordinating residues and 'Inhibitor' refers to the zinc bound inhibitor SAHA. 


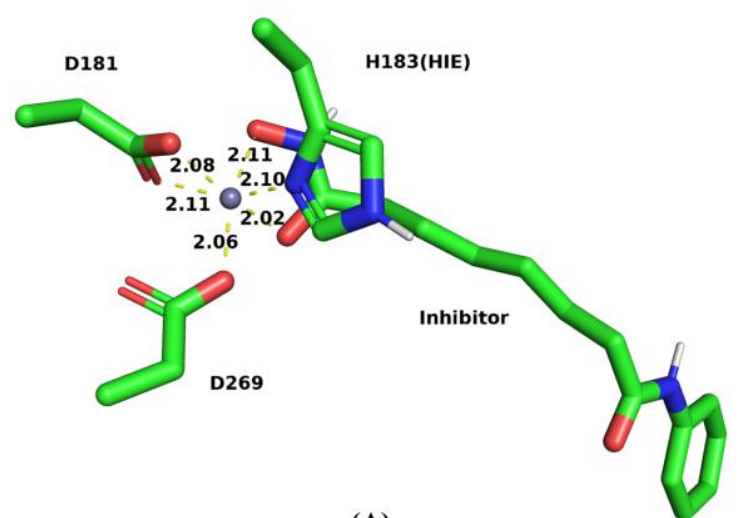

(A)

LJ12-6-4-R

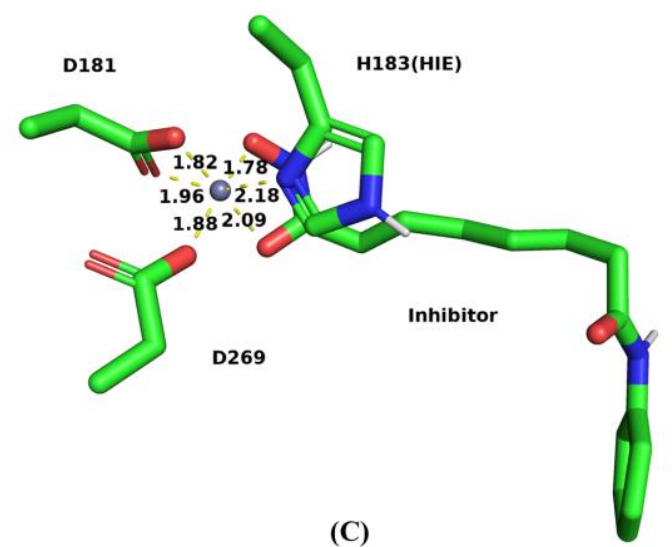

LJ12-6-R

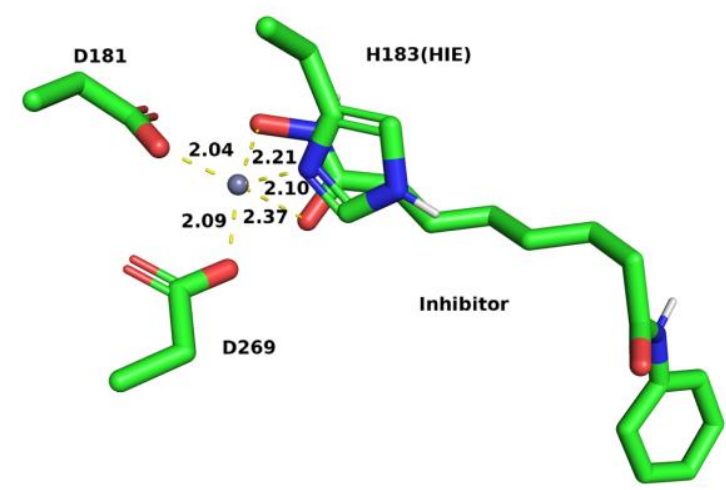

(E)

DFTB3

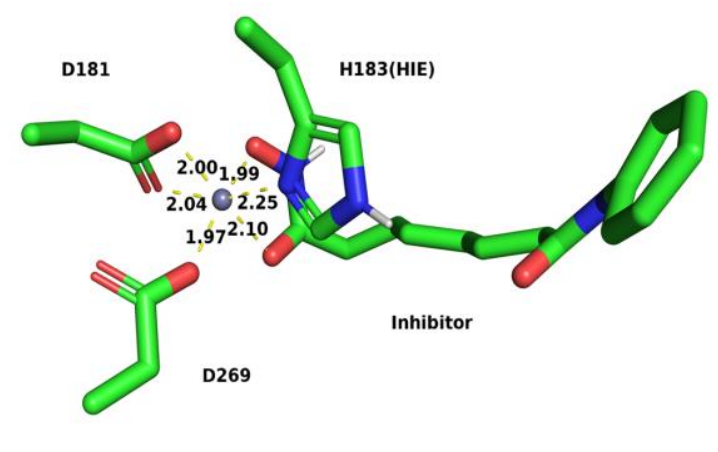

(B)

LJ12-6-4

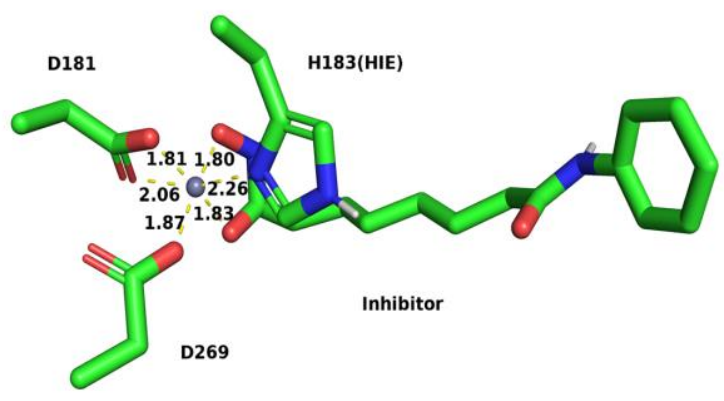

(D)

LJ12-6

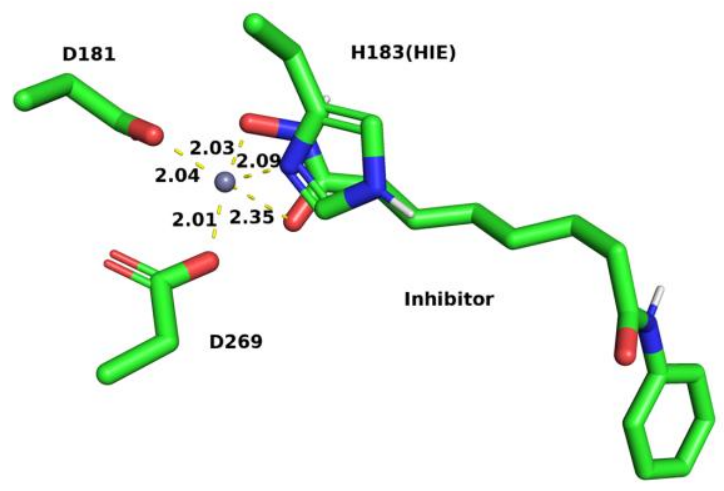

(F)

B3LYP

Figure S8. Representative zinc site geometries of the HDAC2:SAHA complex after MM MD simulations and QM optimization. (A-D) Representative zinc geometries of the four non-bonded models after 100ns MM MD simulation. (E) The representative zinc geometry after 100 ps DFTB3/MM MD simulation. Note restoration of mono-dentate zinc coordination by Asp 181. (F) Representative zinc geometry after B3LYP-D3BJ based QM/MM geometry optimization. 


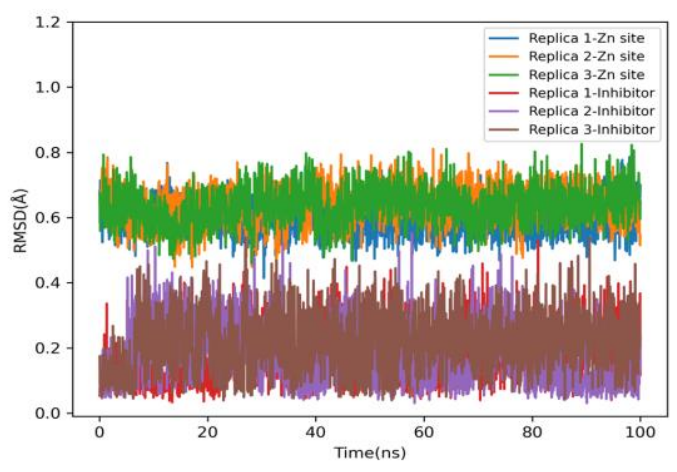

(A)

LJ12-6-4-R

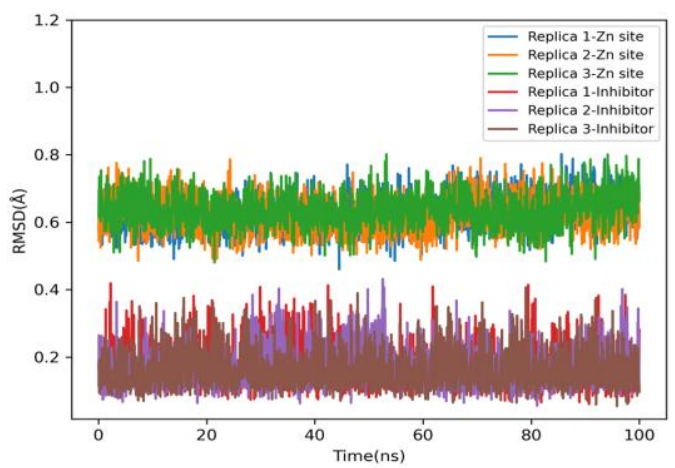

(C)

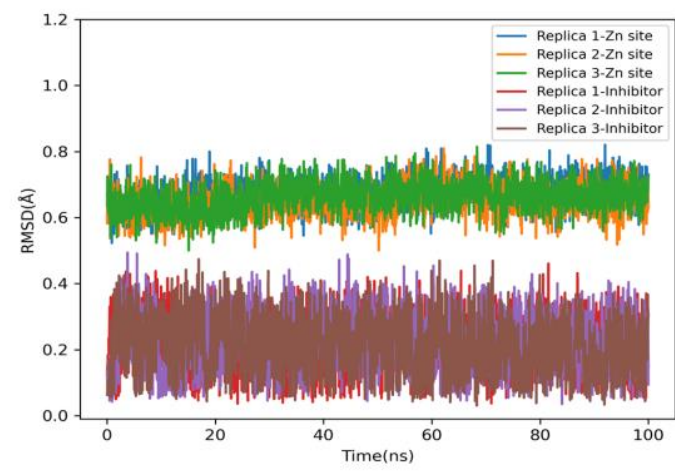

(B)

LJ12-6-4

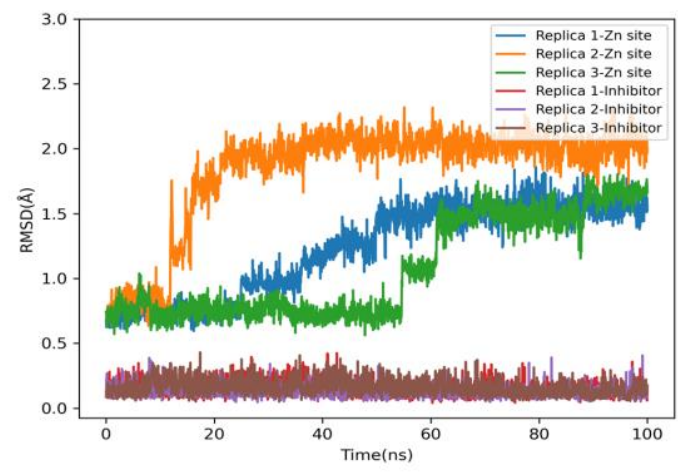

(D)

Figure S9. Time-dependence of RMSD values compared to the crystal structure for MM MD simulations of the IMP1:TGA complex using non-bonded models. Three replicate simulations were performed for each model and each replicate is $100 \mathrm{~ns}$. The 'Zn site' refers to zinc ions and zinc coordinating residues and 'Inhibitor' refers to the zinc bound compound (TGA). 


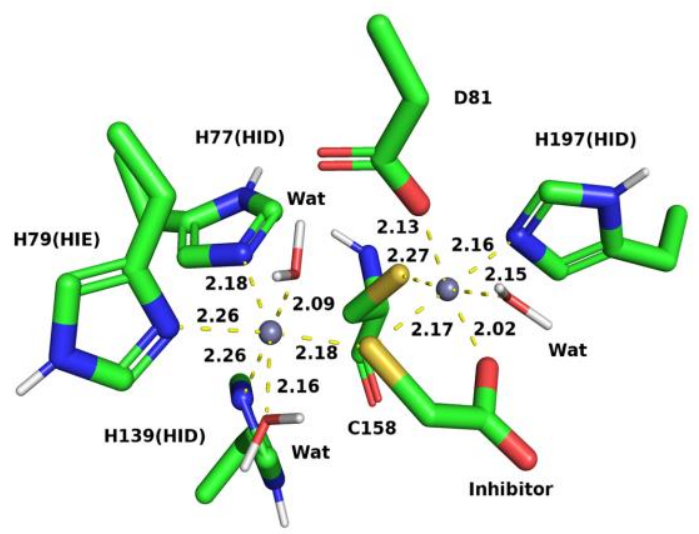

(A)

LJ12-6-4-R

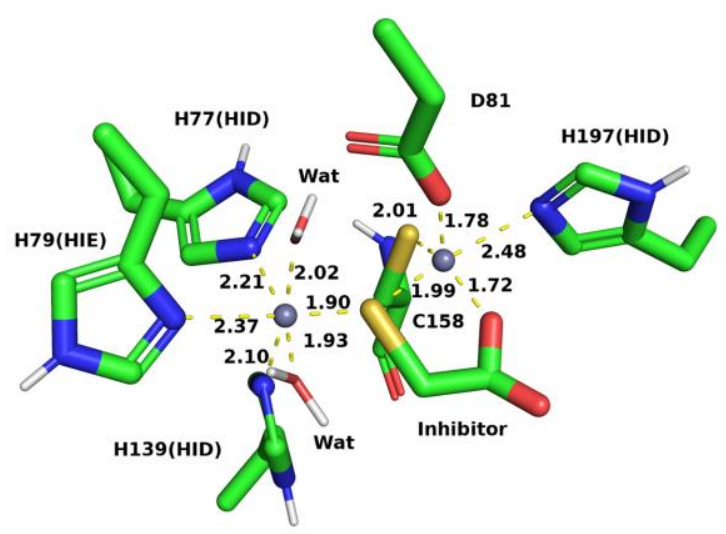

(C)

LJ12-6-R

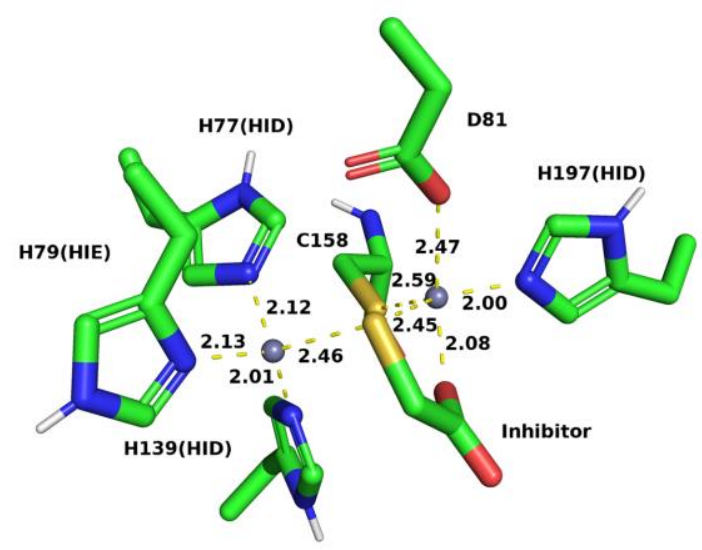

(E)

DFTB3

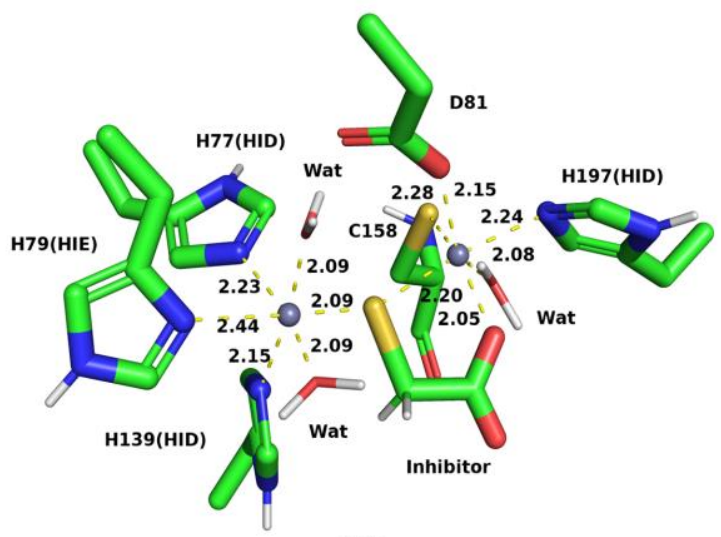

(B)

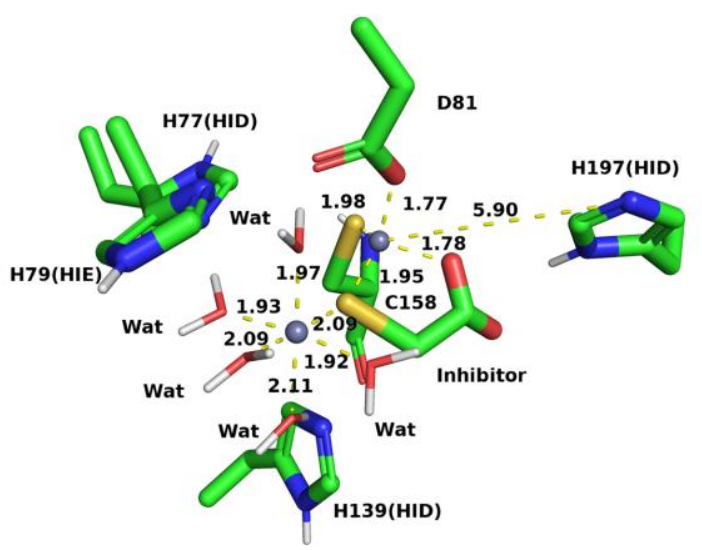

(D)

LJ12-6

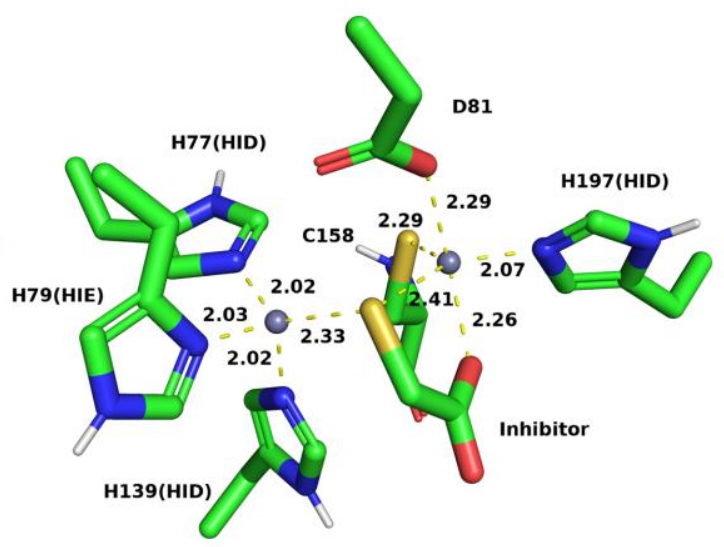

(F)

B3LYP

Figure S10. Representative zinc site geometries of the IMP1:TGA complex after MM MD simulations and QM optimization. (A - D) The representative zinc geometry of the four non-bonded models after 100ns MM MD simulation. (E) The representative zinc geometry after 100 ps DFTB3/MM MD simulation. The extra water molecule was removed. (F) The representative zinc geometry after B3LYP-D3BJ based QM/MM geometry optimization. 


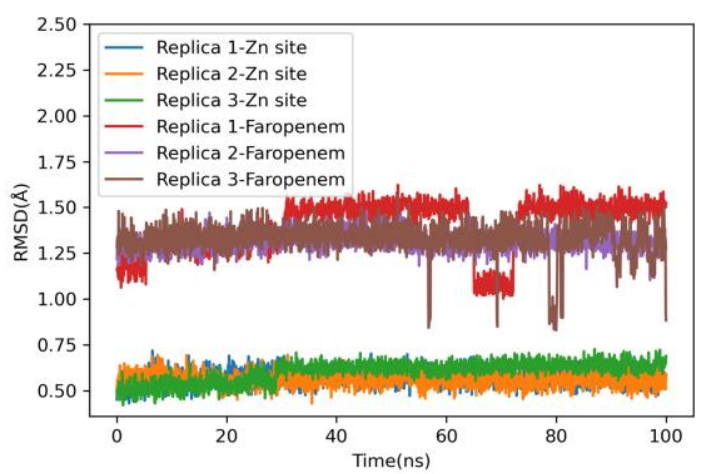

(A)

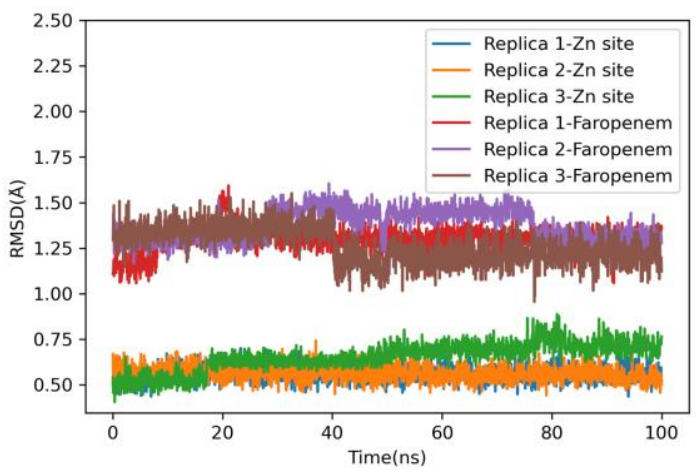

(B)

LJ12-6-4-R

LJ12-6-4

Figure S11. Time-dependence of RMSD values compared to the crystal structure for MM MD simulations of the L1:hydrolyzed faropenem complex using LJ12-6-4 non-bonded models. Three replicate simulations were performed for each model and each replicate is $100 \mathrm{~ns}$. The ' $\mathrm{Zn}$ site' refers to zinc ions and zinc coordinating residues. 


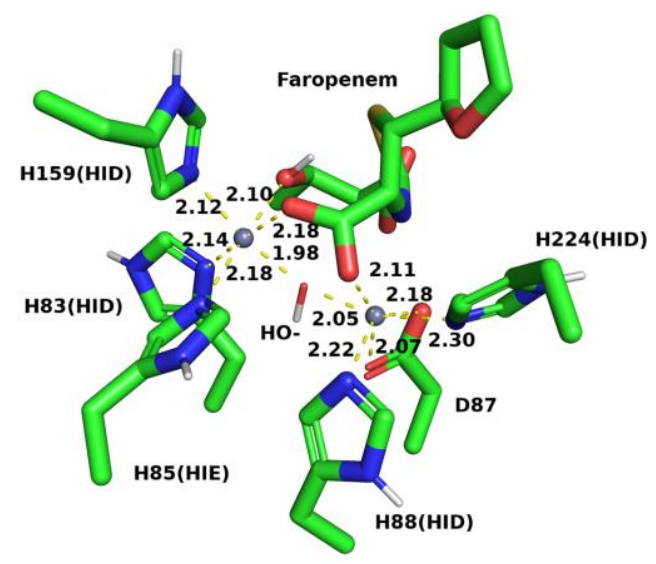

(A)

LJ12-6-4-R

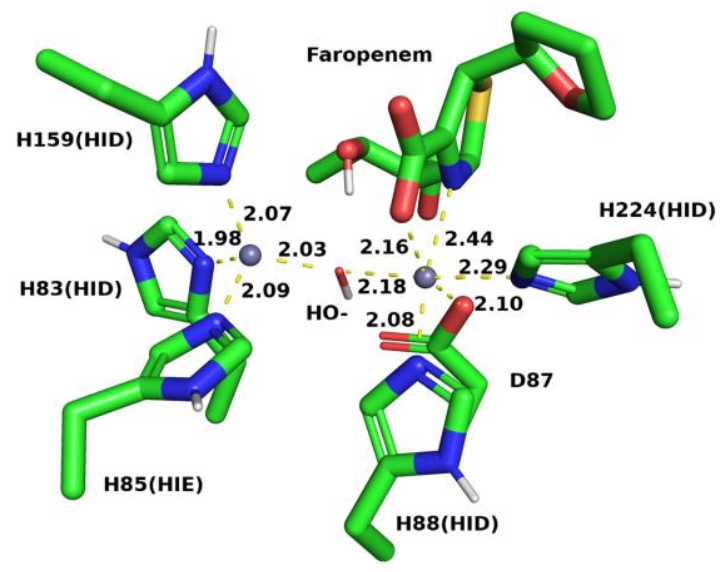

(C)

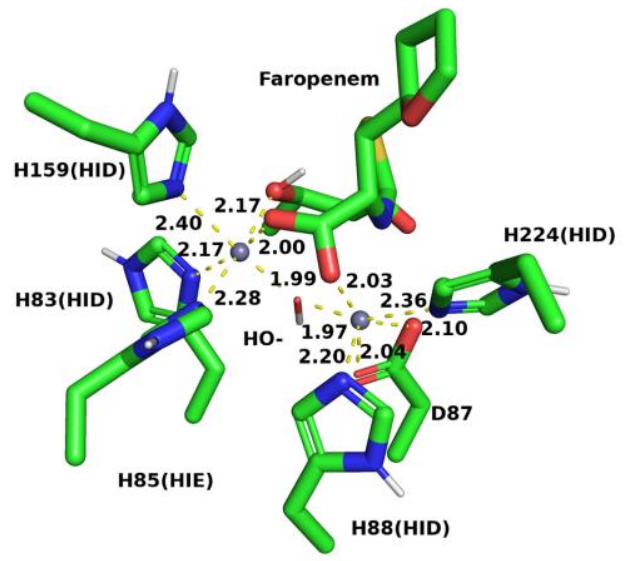

(B)

LJ12-6-4

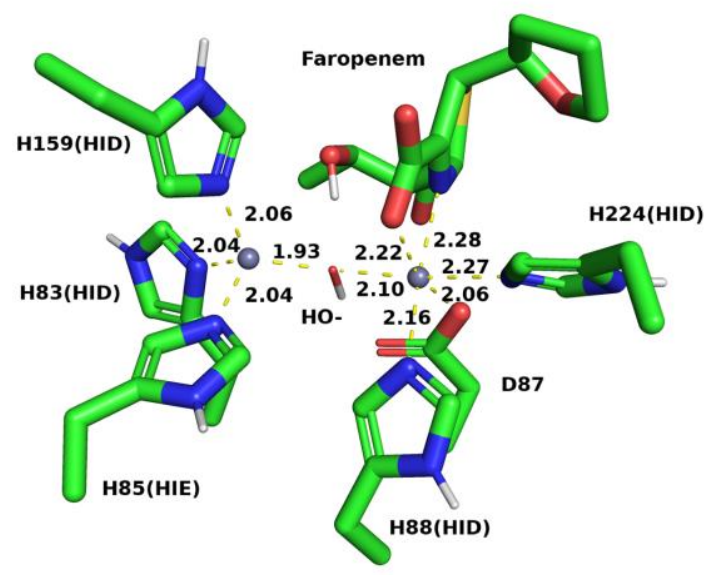

(D)

DFTB3

B3LYP

Figure S12. Representative zinc site geometries of the L1:hydrolyzed faropenem complex after MM MD simulations and QM optimization. (A-B) Representative zinc geometries for L1 complexes with hydrolyzed faropenem during MM MD simulations using LJ12-6-4 L1 non-bonded models with (A) and without (B) restraints, respectively. (C) Representative zinc geometry after 100 ps DFTB3/MM MD simulation. (D) The representative zinc geometry after B3LYP-D3BJ based QM/MM geometry optimization. 

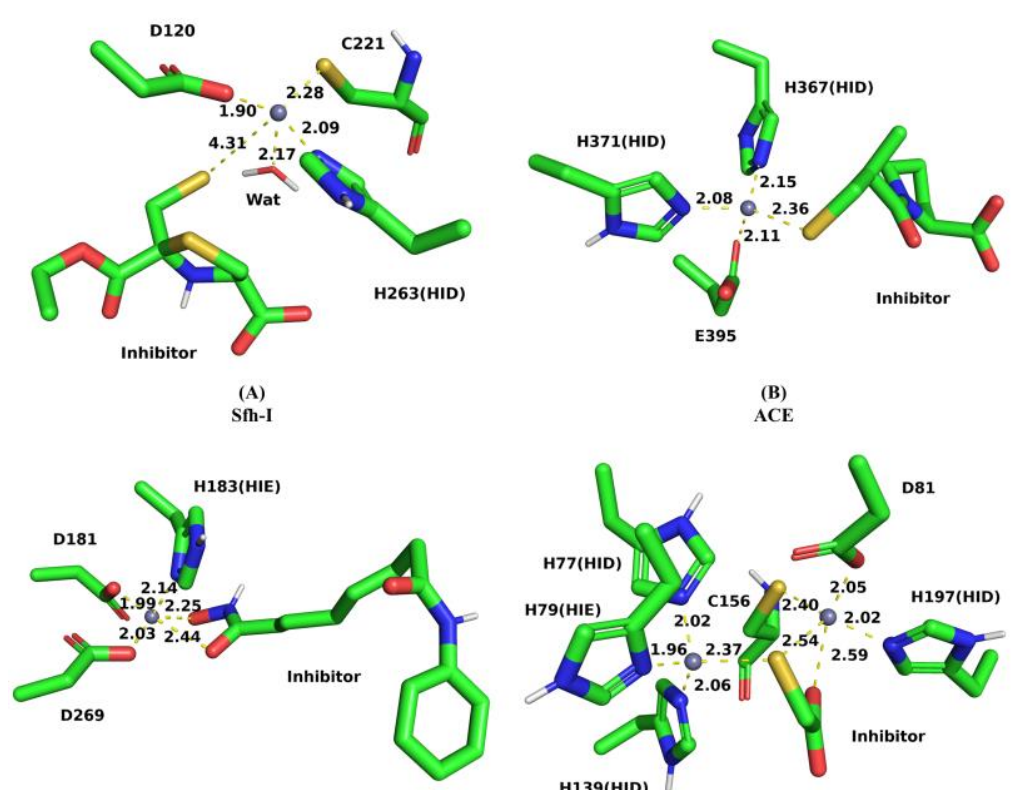

(D)
(B)

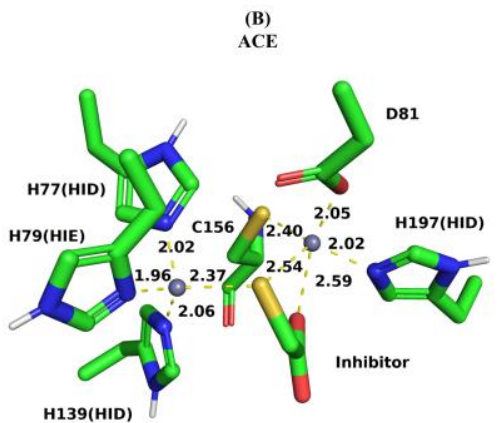

(E)
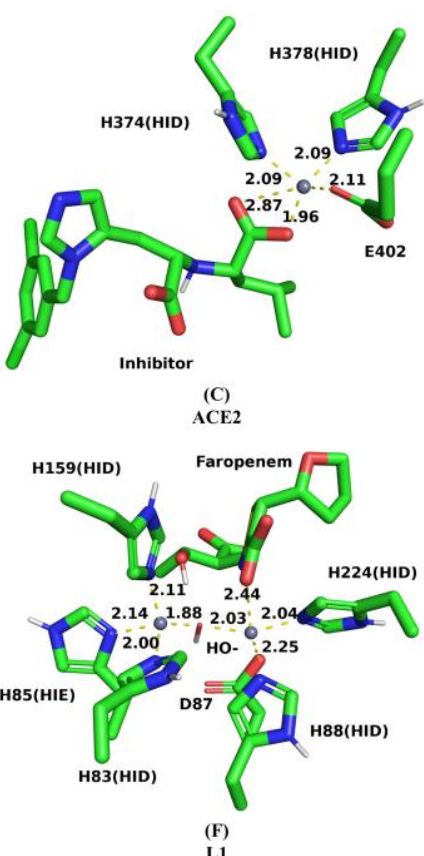

Figure S13. Outcome of DFTB3/MM MD run directly from crystal structures. Representative zinc site geometries of each protein-ligand complex after 2 ns DFTB3/MM MD simulation. Carbon atoms are in green, nitrogen atoms are in blue, oxygen atoms are in red and sulfur atoms are in dark yellow, zinc ions are represented as gray balls. 
Table S1. Zn coordination distance (Å) of four non-bonded MM models for Sfh-I: L-anti-1a complex.

\begin{tabular}{|c|c|c|c|c|c|c|}
\hline & $\begin{array}{l}\text { Zn } \\
\text { Coordinating } \\
\text { Atom }\end{array}$ & LJ126 & LJ126-R & LJ1264 & LJ1264-R & Crystal \\
\hline \multirow{4}{*}{$\begin{array}{l}\text { Mean Coordination } \\
\text { Distance }(\AA)\end{array}$} & Asp120-OD2 & 1.79 & 1.80 & 2.02 & 2.02 & 1.90 \\
\hline & Cys221-SG & 1.99 & 2.03 & 2.20 & 2.28 & 2.37 \\
\hline & His263-NE2 & 3.73 & 2.44 & 2.29 & 2.23 & 2.10 \\
\hline & Inhibitor-S01 & 1.97 & 1.95 & 2.24 & 2.21 & 2.28 \\
\hline Mean CN & & 5.0 & 4.7 & 5.7 & 6.0 & 4.0 \\
\hline \multirow{4}{*}{ Mean Deviation } & Asp120-OD2 & -0.11 & -0.10 & 0.12 & 0.12 & \\
\hline & Cys221-SG & -0.38 & -0.34 & -0.17 & -0.09 & \\
\hline & His263-NE2 & 1.63 & 0.34 & 0.19 & 0.13 & \\
\hline & Inhibitor-S01 & -0.31 & -0.33 & -0.04 & -0.07 & \\
\hline TAD & & 2.43 & 1.11 & 0.53 & 0.41 & \\
\hline TAD per ligand & & 0.61 & 0.28 & 0.13 & 0.10 & \\
\hline Standard Error of TAD & & 1.16 & 1.24 & 0.06 & 0.47 & \\
\hline \multirow{4}{*}{ MPD } & Inhibitor-S01 & $-5.6 \%$ & $-5.4 \%$ & $6.5 \%$ & $6.3 \%$ & \\
\hline & Asp120-0D2 & $-16.0 \%$ & $-14.5 \%$ & $-7.0 \%$ & $-3.8 \%$ & \\
\hline & Cys221-SG & $77.6 \%$ & $16.0 \%$ & $9.2 \%$ & $6.2 \%$ & \\
\hline & His263-NE2 & $-13.6 \%$ & $-14.5 \%$ & $-1.9 \%$ & $-3.2 \%$ & \\
\hline Total Absolute MPD & & $112.9 \%$ & $50.4 \%$ & $24.6 \%$ & $19.5 \%$ & \\
\hline
\end{tabular}

Deviation $(\AA)$ = simulation predicted value - crystal value; $C N=$ Zn Coordination Number; TAD $(\AA)$ = Total Absolute Mean Deviation; MPD =Mean Percentage Deviation. The values presented are averages of the last frames of the three independent MM simulations.

Note: Additional $\mathrm{Zn}^{2+}$ coordinating atoms introduced during simulations that are not presented in the crystal structure are not reported in the table, but are included when calculating the mean coordination number. 
Table S2. Coordination distances between the zinc ion and its coordinating atoms for QM/MM simulations of the Sfh-I:L-anti-1a complex.

\begin{tabular}{|c|c|c|c|c|c|c|c|c|}
\hline & & Action 1 & & & & & DFTB3 & \\
\hline & & Action 2 & & DFTB3 & DFTB3 & DFTB3 & $\begin{array}{c}\text { 6-31G*/ } \\
\text { GD3BJ }\end{array}$ & \\
\hline & $\begin{array}{l}\text { Zn } \\
\text { Coordinating } \\
\text { Atom }\end{array}$ & Action 3 & DFTB3 & 6-31G* & $6-31+G^{*}$ & $\begin{array}{c}\text { 6-31G*/ } \\
\text { GD3BJ }\end{array}$ & $\begin{array}{c}\text { 6-31+G*/ } \\
\text { GD3BJ }\end{array}$ & Crystal \\
\hline & & $\begin{array}{l}\text { Steps of } \\
\text { Action } 3\end{array}$ & & 250 steps & 235 steps $^{[a]}$ & 250 steps & 250 steps & \\
\hline & Inhibitor-S01 & & 2.46 & 2.40 & 2.41 & 2.33 & 2.34 & 2.28 \\
\hline Coordination & Asp120-0D2 & & 1.89 & 1.95 & 2.01 & 1.94 & 1.99 & 1.90 \\
\hline Distance (A) & Cys221-SG & & 2.58 & 2.49 & 2.43 & 2.46 & 2.39 & 2.37 \\
\hline & His263-NE2 & & 2.13 & 2.10 & 2.12 & 2.04 & 2.09 & 2.10 \\
\hline & Inhibitor-S01 & & 0.18 & 0.12 & 0.13 & 0.05 & 0.06 & \\
\hline & Asp120-0D2 & & -0.01 & 0.05 & 0.11 & 0.04 & 0.09 & \\
\hline Deviation & Cys221-SG & & 0.21 & 0.12 & 0.06 & 0.09 & 0.02 & \\
\hline & His263-NE2 & & 0.03 & 0.00 & 0.02 & -0.06 & -0.01 & \\
\hline & Total Absolute & & 0.43 & 0.29 & 0.32 & 0.24 & 0.18 & \\
\hline & Inhibitor-S01 & & $7.9 \%$ & $5.3 \%$ & $5.7 \%$ & $2.2 \%$ & $2.6 \%$ & \\
\hline & Asp120-0D2 & & $-0.5 \%$ & $2.6 \%$ & $5.8 \%$ & $2.1 \%$ & $4.7 \%$ & \\
\hline $\begin{array}{l}\text { Percentage } \\
\text { Deviation }\end{array}$ & Cys221-SG & & $8.9 \%$ & $5.1 \%$ & $2.5 \%$ & $3.8 \%$ & $0.8 \%$ & \\
\hline & His263-NE2 & & $1.4 \%$ & $0.0 \%$ & $1.0 \%$ & $-2.9 \%$ & $-0.5 \%$ & \\
\hline & Total Absolute & & $18.7 \%$ & $13.0 \%$ & $15.0 \%$ & $11.0 \%$ & $8.7 \%$ & \\
\hline Total Absolute & eviation per lig & & 0.11 & 0.07 & 0.08 & 0.06 & 0.05 & \\
\hline Total Absolute & eviation per lig & $1(\%)$ & $4.7 \%$ & $3.2 \%$ & $3.7 \%$ & $2.7 \%$ & $2.2 \%$ & \\
\hline
\end{tabular}

Deviation $(\AA)$ = simulation predicted value - crystal value; $\mathrm{CN}=\mathrm{Zn}$ Coordination Number; $\operatorname{TAD}(\AA)=$ Total Absolute Mean Deviation. DFTB3 refers to 100ps DFTB3/MM MD; 6-31G* and 6-31+G* refer to the basis set used in BL3YP based QM/MM geometry optimization; '+' in a basis set represents the diffuse function; GD3BJ is the empirical dispersion correction.

[a] The geometry optimization has reached the convergency criteria. 
Table S3. Atomic distances $(\AA)$ between the zinc ions and the zinc coordinating atoms for simulations of the ACE inhibitor complex model.

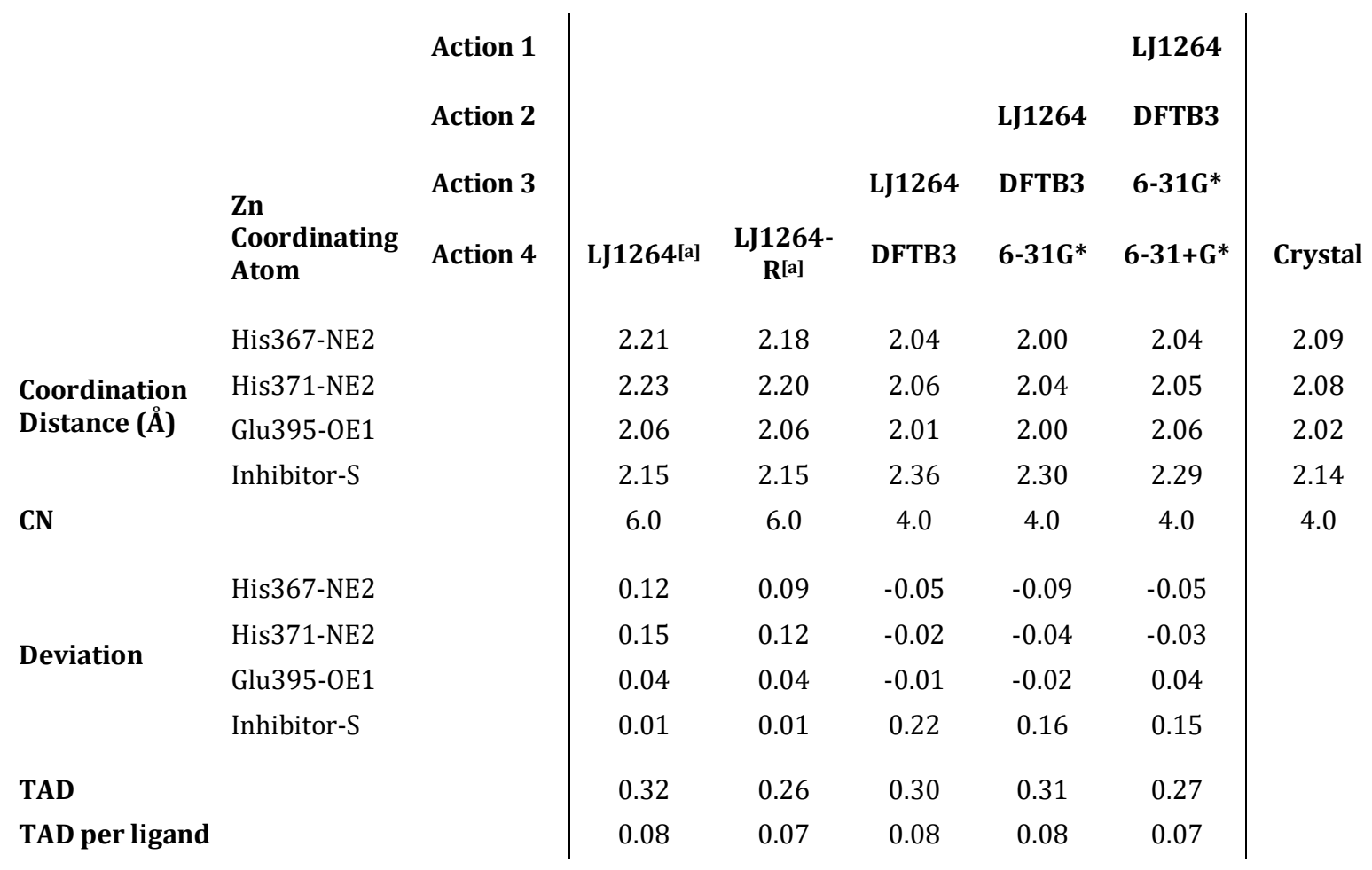

Deviation $(\AA ̊)$ = simulation predicted value - crystal value; $\mathrm{CN}=\mathrm{Zn}$ Coordination Number; $\operatorname{TAD}(\AA \AA)=$ Total Absolute Mean Deviation. DFTB3 refers to 100ps DFTB3/MM MD; 6-31G* and 6-31+G* refer to the basis set used in BL3YPD3BJ based QM/MM geometry optimization.

Note: Additional $\mathrm{Zn}^{2+}$ coordinating atoms introduced during simulations that are not presented in the crystal structure are not reported in the table, but are included when calculating the mean coordination number.

[a] The values presented are averages of the last frames of the three independent MM simulations. 
Table S4. Atomic distances $(\AA)$ between the zinc ions and the zinc coordinating atoms for simulations of the ACE2 inhibitor complex model.

\begin{tabular}{|c|c|c|c|c|c|c|c|c|}
\hline & & Action 1 & & & & & LJ1264 & \\
\hline & & Action 2 & & & & LJ1264 & DFTB3 & \\
\hline & & Action 3 & & & LJ1264 & DFTB3 & 6-31G* & \\
\hline & $\begin{array}{l}\text { Coordinating } \\
\text { Atom }\end{array}$ & Action 4 & LJ1264[a] & $\begin{array}{l}\text { LJ1264- } \\
R^{[a]}\end{array}$ & DFTB3 & 6-31G* & $\begin{array}{l}\text { 6- } \\
31+G^{*}\end{array}$ & Crystal \\
\hline & His374-NE2 & & 2.26 & 2.29 & 2.01 & 1.99 & 2.00 & 2.05 \\
\hline Coordination & His378-NE2 & & 2.26 & 2.22 & 1.99 & 2.11 & 2.13 & 2.31 \\
\hline Distance $(\mathbf{A})$ & Glu402-OE1 & & 2.09 & 2.09 & 2.04 & 1.99 & 2.00 & 2.05 \\
\hline & Inhibitor-01 & & 2.03 & 2.01 & 1.98 & 1.94 & 1.96 & 2.04 \\
\hline CN & & & 6.0 & 6.0 & 4.0 & 4.0 & 4.0 & 4.0 \\
\hline & His374-NE2 & & 0.21 & 0.24 & -0.04 & -0.06 & -0.05 & \\
\hline Devintion & His378-NE2 & & -0.05 & -0.09 & -0.32 & -0.20 & -0.18 & \\
\hline & Glu402-OE1 & & 0.04 & 0.04 & -0.01 & -0.06 & -0.05 & \\
\hline & Inhibitor-01 & & -0.01 & -0.03 & -0.06 & -0.10 & -0.08 & \\
\hline TAD & & & 0.31 & 0.40 & 0.43 & 0.42 & 0.36 & \\
\hline TAD per ligand & & & 0.08 & 0.10 & 0.11 & 0.11 & 0.09 & \\
\hline
\end{tabular}

Deviation $(\AA ̊)$ = simulation predicted value - crystal value; $\mathrm{CN}=\mathrm{Zn}$ Coordination Number; $\operatorname{TAD}(\AA \AA)=$ Total Absolute Mean Deviation. DFTB3 refers to 100ps DFTB3/MM MD; 6-31G* and 6-31+G* refer to the basis set used in BL3YPD3BJ based QM/MM geometry optimization.

Note: Additional $\mathrm{Zn}^{2+}$ coordinating atoms introduced during simulations that are not presented in the crystal structure are not reported in the table, but are included when calculating the mean coordination number.

[a] The values presented are averages of the last frames of the three independent MM simulations. 
Table S5. Zn coordination distance (Å) of four non-bonded MM models for the HDAC-2:SAHA complex.

\begin{tabular}{|c|c|c|c|c|c|c|}
\hline & $\begin{array}{l}\text { Zn } \\
\text { Coordinating } \\
\text { Atom }\end{array}$ & LJ126 & LJ126-R & LJ1264 & LJ1264-R & Crystal \\
\hline \multirow{5}{*}{$\begin{array}{l}\text { Mean Coordination } \\
\text { Distance }(\AA)\end{array}$} & Asp181-OD2 & 2.01 & 1.85 & 2.05 & 2.04 & 2.00 \\
\hline & His183-ND1 & 2.18 & 2.16 & 2.25 & 2.18 & 2.07 \\
\hline & Asp269-0D2 & 1.85 & 1.84 & 2.01 & 2.00 & 1.90 \\
\hline & Inhibitor-01 & 1.82 & 1.82 & 2.02 & 2.07 & 1.96 \\
\hline & Inhibitor-02 & 1.94 & 2.04 & 2.10 & 2.10 & 2.33 \\
\hline Mean CN & & 6.0 & 6.0 & 6.0 & 6.0 & 5.0 \\
\hline \multirow{5}{*}{ Mean Deviation } & Asp181-OD2 & 0.01 & -0.15 & 0.05 & 0.04 & \\
\hline & His183-ND1 & 0.11 & 0.09 & 0.18 & 0.11 & \\
\hline & Asp269-0D2 & -0.05 & -0.06 & 0.11 & 0.10 & \\
\hline & Inhibitor-01 & -0.14 & -0.14 & 0.06 & 0.11 & \\
\hline & Inhibitor-02 & -0.39 & -0.29 & -0.23 & -0.23 & \\
\hline TAD & & 0.70 & 0.73 & 0.63 & 0.58 & \\
\hline TAD per ligand & & 0.14 & 0.15 & 0.13 & 0.12 & \\
\hline Standard Error of TAD & & 0.06 & 0.04 & 0.02 & 0.05 & \\
\hline \multirow{5}{*}{ MPD } & Asp181-OD2 & $0.5 \%$ & $-7.7 \%$ & $2.7 \%$ & $2.2 \%$ & \\
\hline & His183-ND1 & $5.2 \%$ & $4.3 \%$ & $8.5 \%$ & $5.2 \%$ & \\
\hline & Asp269-0D2 & $-2.8 \%$ & $-3.3 \%$ & $5.6 \%$ & $5.3 \%$ & \\
\hline & Inhibitor-01 & $-7.0 \%$ & $-7.0 \%$ & $3.1 \%$ & $5.4 \%$ & \\
\hline & Inhibitor-02 & $16.7 \%$ & $-12.3 \%$ & $-9.9 \%$ & $-9.7 \%$ & \\
\hline Total Absolute MPD & & $32.2 \%$ & $34.6 \%$ & $29.7 \%$ & $27.8 \%$ & \\
\hline
\end{tabular}

Deviation $(\AA)$ = simulation predicted value - crystal value; Percentage Deviation = Deviation value / crystal value; $\mathrm{CN}=$ Zn Coordination Number; TAD $(\AA)=$ Total Absolute Mean Deviation; MPD = Mean Percentage Deviation.

Note: The values presented are averages of the last frames of the three independent MM simulations. Additional $\mathrm{Zn}^{2+}$ coordinating atoms introduced during simulations that are not presented in the crystal structure are not reported in the table, but are included when calculating the mean coordination number. 
Table S6. QM/MM optimized distances $(\AA ̊)$ between the zinc ion and its coordinating atoms for simulations of the HDAC2:SAHA complex.

\begin{tabular}{|c|c|c|c|c|c|c|c|}
\hline & & Action 1 & & & DFTB3 & & \\
\hline & & Action 2 & & DFTB3 & $\begin{array}{c}250 \\
\text { steps } \\
6-31 G^{*}\end{array}$ & DFTB3 & \\
\hline & $\begin{array}{l}\text { Zn } \\
\text { Coordinating } \\
\text { Atoms }\end{array}$ & Action 3 & DFTB3 & 6-31G* & 6-31+G* & 6-31G* & Crystal \\
\hline & & $\begin{array}{l}\text { Steps of } \\
\text { Action } 3\end{array}$ & $100 \mathrm{ps}$ & $\begin{array}{c}250 \\
\text { steps }\end{array}$ & $\begin{array}{c}250 \\
\text { steps }\end{array}$ & $\begin{array}{c}542 \\
\text { steps[a] }\end{array}$ & \\
\hline & Asp181-OD2 & & 2.04 & 1.99 & 2.04 & 1.99 & 2.00 \\
\hline & His183-ND1 & & 2.10 & 2.08 & 2.09 & 2.07 & 2.07 \\
\hline $\begin{array}{l}\text { Coordination } \\
\text { Distance (Å) }\end{array}$ & Asp269-OD2 & & 2.09 & 1.98 & 2.00 & 2.01 & 1.90 \\
\hline & Inhibitor-01 & & 2.21 & 1.99 & 2.03 & 1.99 & 1.96 \\
\hline & Inhibitor-02 & & 2.37 & 2.40 & 2.35 & 2.35 & 2.33 \\
\hline CN & & & 5.0 & 5.0 & 5.0 & 5.0 & 5.0 \\
\hline & Asp181-OD2 & & 0.04 & -0.01 & 0.04 & -0.01 & \\
\hline & His183-ND1 & & 0.03 & 0.01 & 0.02 & 0.00 & \\
\hline Deviation & Asp269-0D2 & & 0.19 & 0.08 & 0.10 & 0.11 & \\
\hline & Inhibitor-01 & & 0.25 & 0.03 & 0.07 & 0.03 & \\
\hline & Inhibitor-02 & & 0.04 & 0.07 & 0.02 & 0.02 & \\
\hline TAD & & & 0.55 & 0.20 & 0.25 & 0.17 & \\
\hline TAD per liganc & & & 0.11 & 0.04 & 0.05 & 0.03 & \\
\hline
\end{tabular}

Deviation $(\AA)$ = simulation predicted value - crystal value; CN = Zn Coordination Number. Total Absolute Mean Deviation. DFTB3 refers to 100ps DFTB3/MM MD; 6-31G* and 6-31+G* refer to the basis set used in BL3YP-D3BJ based QM/MM geometry optimization.

[a] The geometry optimization has reached the convergency criteria. 
Table S7. Zn coordination distance ( $\AA$ ) of four non-bonded MM models for simulations of the IMP1:TGA complex.

\begin{tabular}{|c|c|c|c|c|c|c|c|}
\hline \multicolumn{2}{|c|}{ Atom-1 } & Atom-2 & LJ126 & LJ126-R & LJ1264 & LJ1264-R & Crystal \\
\hline & His77-NE2 & Zn301 & 4.24 & 2.24 & 2.21 & 2.17 & 2.06 \\
\hline & His79-ND1 & Zn301 & 4.31 & 2.30 & 2.29 & 2.24 & 2.05 \\
\hline & His139-NE2 & Zn301 & 4.70 & 2.09 & 2.15 & 2.26 & 2.04 \\
\hline & Inhibitor-S2 & Zn301 & 2.05 & 1.95 & 2.16 & 2.17 & 2.22 \\
\hline \multirow{6}{*}{$\begin{array}{l}\text { Mean Atomic } \\
\text { Distance }(\AA ̊)\end{array}$} & Asp81-0D2 & Zn302 & 1.73 & 1.81 & 2.17 & 2.04 & 2.03 \\
\hline & Cys158-SG & Zn302 & 2.00 & 2.04 & 2.24 & 2.24 & 2.28 \\
\hline & His197-NE2 & Zn302 & 6.59 & 2.44 & 2.26 & 2.23 & 2.13 \\
\hline & Inhibitor-S2 & Zn302 & 1.96 & 2.04 & 2.20 & 2.20 & 2.36 \\
\hline & Inhibitor-02 & Zn302 & 1.74 & 1.78 & 2.06 & 2.04 & 2.25 \\
\hline & Zn301 & Zn302 & 3.88 & 3.82 & 4.21 & 4.02 & 3.55 \\
\hline \multirow[t]{6}{*}{ Mean CN } & Zn301 & & 6.0 & 6.0 & 6.0 & 6.0 & 4.0 \\
\hline & Zn302 & & 4.0 & 5.0 & 6.0 & 6.0 & 5.0 \\
\hline & HIS77-NE2 & $\mathrm{Zn} 1$ & 2.18 & 0.18 & 0.15 & 0.11 & \\
\hline & HIS79-ND1 & $\mathrm{Zn} 1$ & 2.26 & 0.25 & 0.24 & 0.19 & \\
\hline & HIS139-NE2 & $\mathrm{Zn} 1$ & 2.66 & 0.05 & 0.11 & 0.22 & \\
\hline & Inhibitor-S2 & $\mathrm{Zn} 1$ & -0.17 & -0.27 & -0.06 & -0.05 & \\
\hline \multirow{6}{*}{$\begin{array}{l}\text { Mean } \\
\text { Deviation }\end{array}$} & & & & & & & \\
\hline & ASP81-OD2 & $\mathrm{Zn} 2$ & -0.30 & -0.22 & 0.14 & 0.01 & \\
\hline & CYS158-SG & $\mathrm{Zn} 2$ & -0.28 & -0.24 & -0.04 & -0.04 & \\
\hline & HIS197-NE2 & $\mathrm{Zn} 2$ & 4.46 & 0.31 & 0.13 & 0.10 & \\
\hline & Inhibitor-S2 & $\mathrm{Zn} 2$ & -0.40 & -0.32 & -0.16 & -0.16 & \\
\hline & Inhibitor-02 & $\mathrm{Zn} 2$ & -0.51 & -0.47 & -0.19 & -0.21 & \\
\hline \multicolumn{3}{|c|}{ Mean Deviation for Zn301 Coordination } & 7.27 & 0.74 & 0.56 & 0.57 & \\
\hline \multicolumn{3}{|c|}{ Mean Deviation for Zn302 Coordination } & 5.96 & 1.56 & 0.67 & 0.52 & \\
\hline \multicolumn{3}{|l|}{ TAD } & 13.23 & 2.30 & 1.23 & 1.09 & \\
\hline \multicolumn{3}{|l|}{ TAD per ligand } & 1.47 & 0.26 & 0.14 & 0.12 & \\
\hline \multicolumn{3}{|c|}{ Standard Error of TAD } & 1.80 & 0.27 & 0.17 & 0.01 & \\
\hline
\end{tabular}

Deviation $(\AA)$ = simulation predicted value - crystal value; $C N=$ Zn Coordination Number; TAD $(\AA)$ = Total Absolute Mean Deviation. The values presented are averages of the last frames of the three independent MM simulations.

Note: Additional $\mathrm{Zn}^{2+}$ coordinating atoms introduced during simulations that are not presented in the crystal structure are not reported in the table, but are included when calculating the mean coordination number. 
Table S8. Representative QM/MM optimized atomic distances ( $\AA$ ) between the zinc ions and the zinc coordinating atoms for simulations of the IMP-1:TGA complex model.

\begin{tabular}{|c|c|c|c|c|c|c|c|c|c|}
\hline & & & Action 1 & & & DFTB3 & & DFTB3 & \\
\hline & & & Action 2 & & DFTB3 & $\begin{array}{c}250 \\
\text { steps } \\
6-31 G^{*}\end{array}$ & DFTB3 & $\begin{array}{c}449 \text { step } \\
\text { 6-31G* }\end{array}$ & \\
\hline & Atom-1 & Atom-2 & Action 3 & DFTB3 & 6-31G* & $6-31+G^{*}$ & 6-31G* & $6-31+G^{*}$ & Crystal \\
\hline & & & $\begin{array}{l}\text { Steps of } \\
\text { Action } 3\end{array}$ & & $\begin{array}{c}250 \\
\text { steps }\end{array}$ & $\begin{array}{c}340 \\
\text { steps }\end{array}$ & $\begin{array}{c}449 \\
\text { steps[a] }\end{array}$ & $\begin{array}{c}250 \\
\text { steps[a] }\end{array}$ & \\
\hline & His77-NE2 & Zn301 & & 2.08 & 2.02 & 2.05 & 2.02 & 2.04 & 2.06 \\
\hline & His79-ND1 & Zn301 & & 2.08 & 2.04 & 2.07 & 2.03 & 2.05 & 2.05 \\
\hline & His139-NE2 & Zn301 & & 2.08 & 2.03 & 2.06 & 2.02 & 2.06 & 2.04 \\
\hline & Inhibitor-S2 & Zn301 & & 2.20 & 2.32 & 2.32 & 2.33 & 2.34 & 2.22 \\
\hline $\begin{array}{l}\text { Atomic } \\
\text { Distance }\end{array}$ & Asp81-0D2 & Zn302 & & 2.36 & 2.27 & 2.16 & 2.29 & 2.18 & 2.03 \\
\hline & Cys158-SG & Zn302 & & 2.43 & 2.30 & 2.34 & 2.29 & 2.29 & 2.28 \\
\hline & His197-NE2 & Zn302 & & 2.01 & 2.06 & 2.08 & 2.07 & 2.10 & 2.13 \\
\hline & Inhibitor-S2 & Zn302 & & 2.43 & 2.41 & 2.43 & 2.41 & 2.41 & 2.36 \\
\hline & $\begin{array}{l}\text { Inhibitor- } \\
02\end{array}$ & Zn302 & & 2.37 & 2.28 & 2.48 & 2.26 & 2.44 & 2.25 \\
\hline & His77-NE2 & Zn301 & & 0.02 & -0.04 & -0.01 & -0.04 & -0.02 & \\
\hline & His79-ND1 & Zn301 & & 0.03 & -0.01 & 0.02 & -0.02 & 0.00 & \\
\hline & His139-NE2 & Zn301 & & 0.04 & -0.01 & 0.02 & -0.02 & 0.02 & \\
\hline & Inhibitor-S2 & Zn301 & & -0.02 & 0.10 & 0.10 & 0.11 & 0.12 & \\
\hline Deviation & Asp81-0D2 & Zn302 & & 0.33 & 0.24 & 0.13 & 0.26 & 0.15 & \\
\hline & Cys158-SG & Zn302 & & 0.15 & 0.02 & 0.06 & 0.01 & 0.01 & \\
\hline & His197-NE2 & Zn302 & & -0.12 & -0.07 & -0.05 & -0.06 & -0.03 & \\
\hline & Inhibitor-S2 & Zn302 & & 0.07 & 0.05 & 0.07 & 0.05 & 0.05 & \\
\hline & $\begin{array}{l}\text { Inhibitor- } \\
02\end{array}$ & Zn302 & & 0.12 & 0.03 & 0.23 & 0.01 & 0.19 & \\
\hline TAD for $\mathrm{Zn}$ & 01 Coordina & & & 0.11 & 0.16 & 0.15 & 0.19 & 0.16 & \\
\hline TAD for $\mathrm{Zn}$ & 02 Coordina & & & 0.79 & 0.41 & 0.54 & 0.39 & 0.43 & \\
\hline TAD per lig & and & & & 0.10 & 0.06 & 0.08 & 0.06 & 0.07 & \\
\hline
\end{tabular}

Deviation $(\AA)$ = simulation predicted value - crystal value; TAD $(\AA)=$ = Total Absolute Mean Deviation. DFTB3 refers to 100ps DFTB3/MM MD; 6-31G* and 6-31+G* refer to the basis set used in BL3YP-D3BJ based QM/MM geometry optimisation.

[a] The geometry optimization has reached the convergency criteria. 
Table S9. Representative atomic distances $(\AA)$ between the zinc ions and the zinc coordinating atoms for simulations of the L1:Faropenem complex model.

\begin{tabular}{|c|c|c|c|c|c|c|c|c|c|}
\hline & & & Action 1 & & & & & LJ1264-R & \\
\hline & & & Action 2 & & & & LJ1264-R & DFTB3 & \\
\hline & & & Action 3 & & & LJ1264-R & DFTB3 & 6-31G* & \\
\hline & Atom-1 & Atom-2 & Action 4 & LJ1264[a] & $\begin{array}{l}\text { LJ1264- } \\
R[a]\end{array}$ & DFTB3 & 6-31G* & $6-31+G^{*}$ & Crystal \\
\hline & HIS83-ND1 & Zn267 & & 2.20 & 2.17 & 1.98 & 2.02 & 2.04 & 2.06 \\
\hline & HIS85-NE2 & Zn267 & & 2.23 & 2.16 & 2.09 & 2.03 & 2.05 & 2.08 \\
\hline & HIS159-NE2 & Zn267 & & 2.29 & 2.15 & 2.07 & 2.04 & 2.06 & 2.05 \\
\hline & HO- & Zn267 & & 1.99 & 1.97 & 2.03 & 1.92 & 1.93 & 1.92 \\
\hline Atomic & HIS88-NE2 & $\mathrm{Zn} 268$ & & 2.18 & 2.27 & 2.08 & 2.15 & 2.16 & 2.15 \\
\hline Distance $(\AA)$ & ASP87-OD2 & Zn268 & & 2.07 & 2.12 & 2.10 & 2.04 & 2.06 & 2.12 \\
\hline & HIS224-NE2 & Zn268 & & 2.29 & 2.29 & 2.29 & 2.29 & 2.27 & 2.11 \\
\hline & HO- & Zn268 & & 2.00 & 2.02 & 2.18 & 2.08 & 2.10 & 2.07 \\
\hline & Faropenem-010 & Zn268 & & 2.53 & 2.48 & 2.16 & 2.12 & 2.22 & 2.22 \\
\hline & Faropenem-N06 & Zn268 & & 2.43 & 2.48 & 2.44 & 2.30 & 2.28 & 2.31 \\
\hline & Zn267 & Zn268 & & 3.68 & 3.74 & 3.95 & 3.87 & 3.90 & 3.57 \\
\hline CN & $\mathrm{Zn} 267$ & & & 6.0 & 6.0 & 4.0 & 4.0 & 4.0 & 4.0 \\
\hline & Zn268 & & & 6.3 & 6.0 & 6.0 & 6.0 & 6.0 & 6.0 \\
\hline & HIS83-ND1 & Zn267 & & 0.14 & 0.11 & -0.08 & -0.04 & -0.02 & \\
\hline & HIS85-NE2 & Zn267 & & 0.15 & 0.08 & 0.01 & -0.05 & -0.03 & \\
\hline & HIS159-NE2 & Zn267 & & 0.24 & 0.10 & 0.02 & -0.01 & 0.01 & \\
\hline & HO- & Zn267 & & 0.07 & 0.05 & 0.11 & 0.00 & 0.01 & \\
\hline Deviation & HIS88-NE2 & $\mathrm{Zn} 268$ & & 0.03 & 0.12 & -0.07 & 0.00 & 0.01 & \\
\hline & ASP87-OD2 & Zn268 & & -0.05 & 0.00 & -0.02 & -0.08 & -0.06 & \\
\hline & HIS224-NE2 & Zn268 & & 0.18 & 0.18 & 0.18 & 0.18 & 0.16 & \\
\hline & HO- & Zn268 & & -0.07 & -0.05 & 0.11 & 0.01 & 0.03 & \\
\hline & Faropenem-010 & Zn268 & & 0.31 & 0.26 & -0.06 & -0.10 & 0.00 & \\
\hline & Faropenem-N06 & Zn268 & & 0.12 & 0.17 & 0.13 & -0.01 & -0.03 & \\
\hline Deviation for & In267 Coordinati & & & 0.60 & 0.34 & 0.22 & 0.10 & 0.07 & \\
\hline Deviation for & n268 Coordinati & & & 0.76 & 0.78 & 0.57 & 0.38 & 0.29 & \\
\hline TAD & & & & 1.35 & 1.12 & 0.79 & 0.48 & 0.36 & \\
\hline TAD/Crystal & gand & & & 0.14 & 0.11 & 0.08 & 0.05 & 0.04 & \\
\hline Standard Err & $r$ of TAD & & & 0.47 & 0.48 & / & / & / & \\
\hline
\end{tabular}


Deviation $(\AA)$ = simulation predicted value - crystal value; CN = Zn Coordination Number; TAD $(\AA)$ = Total Absolute Mean Deviation. DFTB3 refers to 100 ps DFTB3/MM MD; 6-31G* and 6-31+G* refer to the basis set used in BL3YP-D3BJ based QM/MM geometry optimisation.

Note: Additional $\mathrm{Zn}^{2+}$ coordinating atoms introduced during simulations that are not presented in the crystal structure are not reported in the table, but are included when calculating the mean coordination number.

[a] The values presented are averages of the last frames of the three independent MM simulations. 